\title{
Simulating Wavefront Correction via Deformable Mirrors at X-Ray Beamlines
}

T. Pardini, L. A. Poyneer, M. J. Pivovaroff

August 1, 2012

X-ray Adaptive Optics

San Diego, CA, United States

August 14, 2012 through August 14, 2012 
This document was prepared as an account of work sponsored by an agency of the United States government. Neither the United States government nor Lawrence Livermore National Security, LLC, nor any of their employees makes any warranty, expressed or implied, or assumes any legal liability or responsibility for the accuracy, completeness, or usefulness of any information, apparatus, product, or process disclosed, or represents that its use would not infringe privately owned rights. Reference herein to any specific commercial product, process, or service by trade name, trademark, manufacturer, or otherwise does not necessarily constitute or imply its endorsement, recommendation, or favoring by the United States government or Lawrence Livermore National Security, LLC. The views and opinions of authors expressed herein do not necessarily state or reflect those of the United States government or Lawrence Livermore National Security, LLC, and shall not be used for advertising or product endorsement purposes. 


\title{
Simulating Wavefront Correction via Deformable Mirrors at X-Ray Beamlines
}

\author{
Tommaso Pardini ${ }^{a}$, Lisa A. Poyneer ${ }^{a}$, Audrey Plinta ${ }^{b}$, Jeffrey L. Cavaco ${ }^{b}$, Michael J. \\ Pivovaroff ${ }^{a}$ \\ ${ }^{a}$ Lawrence Livermore National Laboratory, Livermore, CA 94550, USA \\ ${ }^{b}$ Northrop Grumman, AOA Xinetics Inc., Devens, MA 01434, USA
}

\begin{abstract}
Deformable mirrors (DMs) have been successfully used in astronomical adaptive optics at visible and near-infrared wavelengths, greatly reducing atmospheric-induced aberrations. Building upon the extensive techniques and methods developed for these applications, we propose to extend this capability to the soft and hard x-ray regime in order to take full advantage of the beam quality characteristic of new facilities such as the National Synchrotron Light Source (NSLS-II), and the Linac Coherent Light Source (LCLS). Achieving this goal challenges both current mirror manufacturing techniques and wavefront propagation modeling. Lawrence Livermore National Laboratory (LLNL), in collaboration with Northrop Grumman AOA Xinetics Inc., is currently developing an x-ray deformable mirror to correct for wave-front aberrations introduced along the beam path of a typical x-ray beamline. To model the expected performance of such a mirror, we have developed a simulation based on the wavefront propagation code PROPER. We will present the current implementation of the software, which models actuation of a deformable mirror and evaluates its effect on wavefront correction.
\end{abstract}

Keywords: Adaptive x-ray optics, deformable mirrors, wavefront correction, wavefront simulations

\section{WAVEFRONT CORRECTION IN THE X-RAY REGIME}

Today's requirements for experiments conducted at x-ray lightsources are more demanding than ever. From Biology to Physics, the most exciting topics currently being investigated require x-ray beams that are intense, well-focused and spatially coherent. That these experiments are even at our reach is due to the current or imminent availability of state-of-the-art facilities such as the Linac Coherent Light Source (LCLS) and the National Synchrotron Light Source II (NSLS II). The LCLS has been active since 2009 and the data in hand demonstrates that its x-ray beam is truly diffraction limited. ${ }^{1,2}$

A major effort undertaken as part of the construction of the LCLS was the design, fabrication and implementation of reflective optics to safely transport the x-ray beam to users while preserving beam brilliance and minimizing disruption of coherence. Lawrence Livermore National Laboratory (LLNL) had the primary responsibility to fabricate two discrete mirror systems: the soft x-ray offset mirrors system (SOMS) consisting of four optical elements working between $\approx 0.5-2 \mathrm{keV}$ and the hard x-ray offset mirror systems (HOMS), consisting of two optical elements working between $2-25 \mathrm{keV}^{3-6}$

Although the mirrors that constitute the SOMS and HOMS represent some of the highest performance mirrors constructed for a lightsource application, there is still need for even improving the quality of the substrates, the limiting factor for these mirrors. These optics were fabricated using traditional fabrication methods and even very small imperfections in the mirror surface at length-scales longer than a few $\mathrm{mm}$ will introduce small wavefront distortions. The use of a deformable mirror offers to greatly minimize residual errors left once the mirror is fabricated. It also offers the promise of changing the shape of the mirror with time. For example, transport optics are generally placed in a harsh x-ray environment which over time can degrade the quality of the optical surface. A deformable mirror is a natural choice to correct for both these effects.

Further author information: (Send correspondence to T. Pardini)

T. Pardini: E-mail: pardini2@llnl.gov, Telephone: 1 925-423-1889 
LLNL, in collaboration with Northrop Grumman AOA Xinetics Inc., is developing the technology needed to successfully deploy a deformable mirror at an x-ray facility. This project will see the design and fabrication of a hard x-ray, 450mm long deformable mirror. Another important area of research is a modeling tool, consisting of custom written software to simulate how a deformable mirror (DM) will effect the wavefront propagation of the beam as it reflects off the mirror surface. This paper focuses on the simulation work, and in particular on how these simulations can help quantify important parameters such as degree of wavefront correction and optimal DM placement location. We postpone the description of the hardware to a later manuscript.

In section 2 we will describe the code we have developed and show how we used it to simulate beam transport to the Far Experimental Hall (FEH) at the LCLS. Our results will be compared to results already published in literature providing a consistency check for the code. In section 3, using the LCLS transport system as template, we discuss the case where the current offset mirrors are replaced by DMs. For the same facility, in section 4 we discuss the option of a standalone downstream DM to correct wavefront aberrations introduced by the preceding offset mirrors. Finally in section 5 we draw our conclusions. The simulations presented in this paper use the LCLS HOMS beam transport system as a template since we have the detailed metrology data on the figure errors of all the mirrors fabricated for this system. It is extremely important to note, however, that our codes and approaches are extensible to any x-ray lightsource beamline of choice.

\section{WAVEFRONT PROPAGATION AT AN X-RAY BEAMLINE: THE LINAC COHERENT LIGHT SOURCE}

The field of adaptive optics applied to the x-ray regime is still in its early stages. Although hardware has been fabricated and tested with success in France and Japan, ${ }^{7,8}$ there is no standardized software or techniques to predict performance. Additionally, limited data is available to predict the performance of a deformable mirror given its manufacturing specifications. At the same time successful deployment of a DM at any x-ray beamline heavily relies on our ability to quantify aspects such as performance dependance on wavelength, figure errors and DM placement location. For this reason, as part of our x-ray adaptive optic project, we have developed code to simulate beam propagation along an x-ray beamline. Such code uses the PROPER ${ }^{9}$ library of functions for wavefront propagation developed by John Krist at the Jet Propulsion Laboratory. The code allows for easy simulation of any beamline of choice with customized source and beam transport mechanism. Its main feature is the capability of introducing DMs along the beamline. These DMs can be used to correct for phase errors that they themselves, or other conventional mirrors introduce. Below we list the input parameters necessary to run the code:

- Source specification:

- Shape

- Size

- Wavelength (monochromatic)

- Beam transport system specification:

- Number of mirrors (flat only, no focusing optics yet)

- For each mirror

* Length and width

* Location with respect to source

* Grazing angle

* Figure error (from file or random)

* Conventional or deformable 


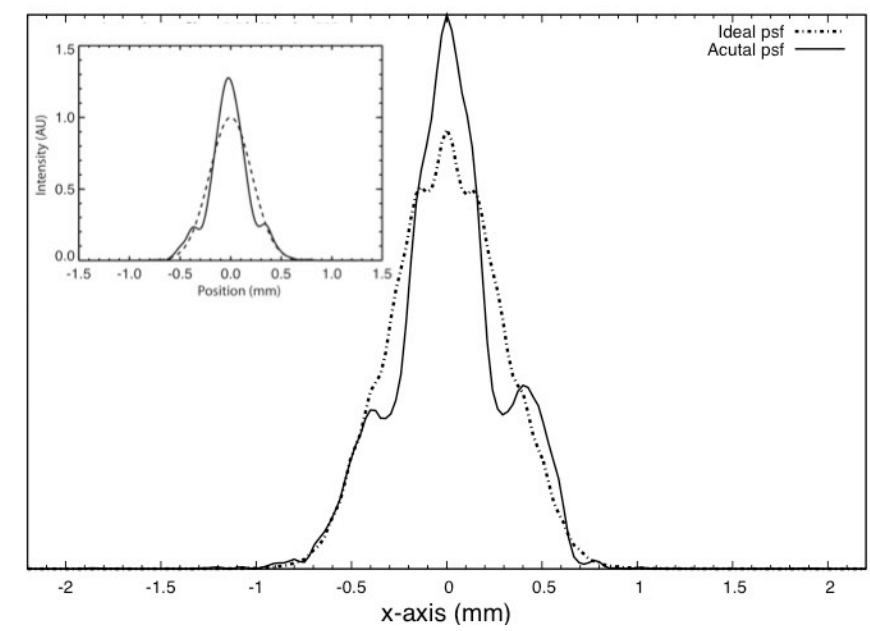

Figure 1. Beam profile at the Far Experimental Hall of the Linac Coherent Light Source (solid line). The diffraction limited psf is also shown with a dash line. (Inset) Same psf calculated by Barty et al. ${ }^{10}$

Every x-ray mirror is different from any other, due to the uniqueness of its figure errors. An important feature of our code is the flexibility in dealing with such figure errors. If data is available for a specific optic, for instance interferometry data, it is possible to include it into the simulation. On the other hand, if the figure error is not known, a random pattern can be generated. Before using our code to study the performance of the DM under development, we ran a simulation of the LCLS HOMS transport system. We then compared the output with published results on beam propagation for the same facility ${ }^{10}$ therefore establishing a consistency check for our code. In the following we described the parameters used for this preliminary simulation.

The $8 \mathrm{keV}$ LCLS beam is modeled as a gaussian beam with a FWHM of $80 \mu \mathrm{m}$. The transport mechanism consists of two offset mirrors, each with a clear aperture $385 \mathrm{~m}$ long and $15 \mathrm{~mm}$ wide, placed $93.9 \mathrm{~m}$ and $105.3 \mathrm{~m}$ away from the undulator exit respectively. The grazing angle for both mirrors is $1.35 \mathrm{mrad}$ and their figure error files are based on interferometry data collected at LLNL during mirror characterization conducted before deployment. ${ }^{4}$ Both mirrors are conventional reflective optics with no deformable capabilities. The FEH hutch is $277.7 \mathrm{~m}$ downstream of the last offset mirror. The output of the code is shown in Fig. 1. The dash line represents the beam profile in the absence of figure error on the offset mirrors. In this case the shoulders at both sides of the central peak are caused by diffraction effects from the mirrors edges. The solid line represents the beam profile when the figure errors for both offset mirrors are introduced. The effect of surface errors are visible in the low intensity wings at either sides of the central peak. This result is consistent with what obtained by Barty et al. ${ }^{10}$ (inset) and proves that our code is working properly. In the next section, once again using the LCLS beam transport system as a template, we show how we could ideally correct for wavefront distortions if we replaced the existing offset mirrors with two DMs, each one with the same specifications as the one we are currently developing.

\section{INTRODUCING A DEFORMABLE MIRROR}

In an ideal scenario replacing all conventional optics with DMs would provide maximum wavefront correction while propagating a beam to the experimental hutch. We would argue that this way no wavefront sensor would be necessary as the DMs would only have to correct for their own figure errors, which could be measured before deployment. This scenario is what we investigate in this section. Once again we use the LCLS hard x-ray transport system as a test subject. The source is the same as in the previous section while the two offset mirrors have been replaced by two DMs. It is important to realized that the two DMs introduced in our simulation are a 
correct representation of the actual mirror we are developing. Besides matching size and actuator locations, this also means that surface actuation is driven by a set of influence functions directly obtained via Finite Element Analysis (FEA) on the mirror model. Therefore the result of our analysis provides direct quantitative insights on future performance of our DM. It should be pointed out however that the actual mirror is not available yet and therefore no data is available on its figure errors. For this reason, in this simulation each DM has been given a random figure error generated by filtering a random surface profile with the power spectral density (PSD) measured on the LCLS offset mirrors. Given the similarity in substrate size and manufacturing process, it is reasonable to expect the DM under development to have similar surface errors as the LCLS offset mirrors.

\section{Beam profile at FEH}
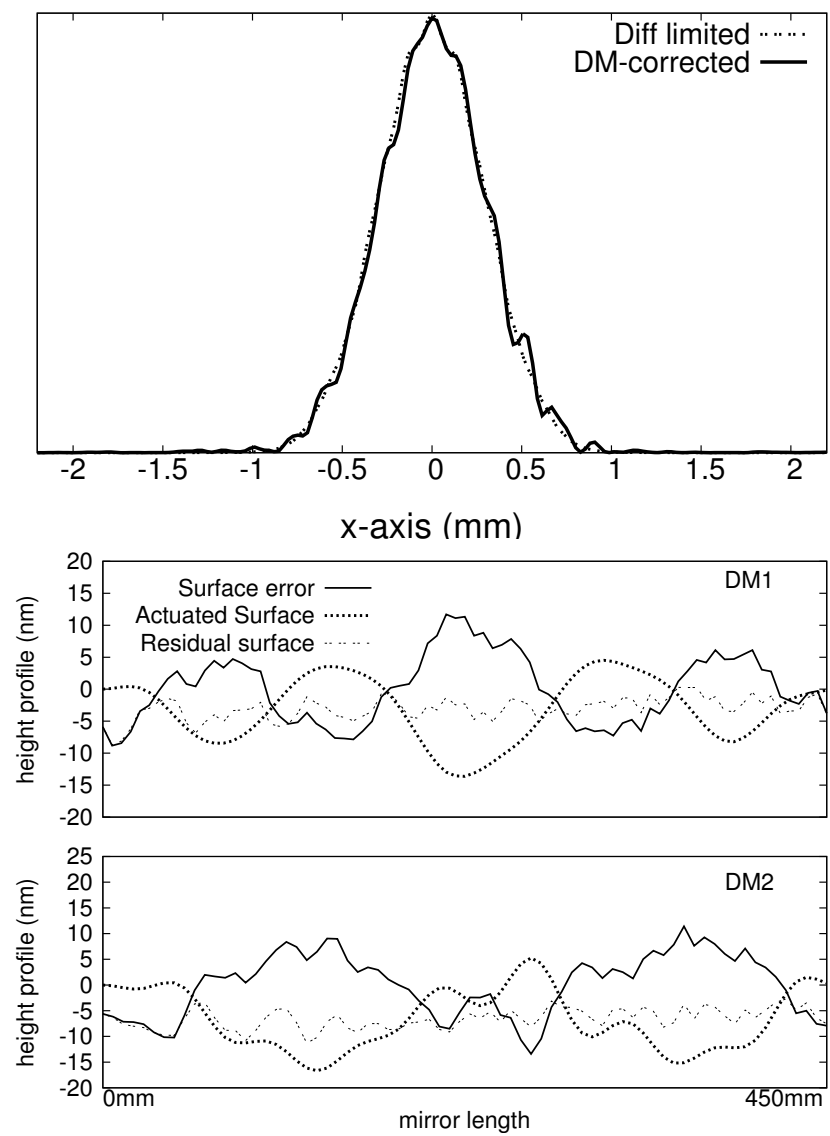

Figure 2. Top Panel: beam profile at LCLS FEH hutch. Diffraction limited and corrected profile are shown. Bottom panel: Figure error, actuated surface and residual surface for the two DMs. Simulation was ran using a $1536 x 1536$ size grid

The results of the simulation are shown in Fig. 2 where we display the DM-corrected beam profile at the FEH (solid line). The diffraction limited profile is also shown (dash line). The impact of wavefront correction via the DMs is evident in the absence of the two low intensity wings at either side of the central peak. The profile is more uniform and closer to the original gaussian shape. As mentioned previously, in this scenario the DMs only need to correct for their own figure errors. To get a closer look at the wavefront correction process we plot the figure error, the actuated surface and the residual surface for each mirror. It is important to remember that the cutoff frequency for the PSD of the random figure error generated in the simulation depends on the sampling used. Here we have used a sampling of $\sim 8 \mu \mathrm{m}$ in the beam reference frame; this translates in a cutoff for the PSD at the highest spatial frequency of $\omega_{\text {cutoff }} \approx 6 \times 10^{-5} \mathrm{~nm}^{-1}$. Considering that DM actuator spacing is $10 \mathrm{~mm}$, the highest frequency we can correct is $\sim 3.7 \times 10^{-5} \mathrm{~nm}^{-1}$. The discrepancy between the diffraction limited and the DM-corrected beam profile in Fig. 2 can be attributed to the range of figure error frequencies we cannot correct 

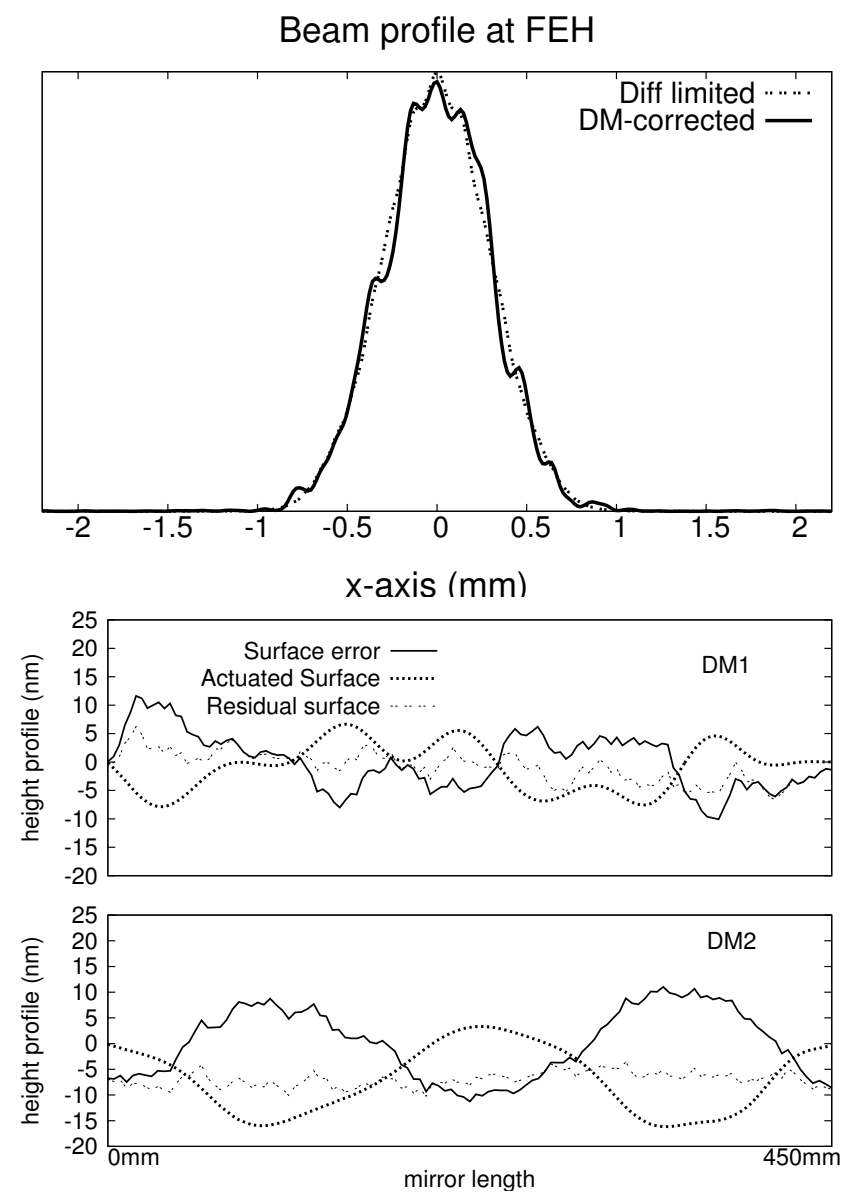

Figure 3. Top Panel: beam profile at LCLS FEH hutch. Diffraction limited and corrected profile are shown. Bottom panel: Figure error, actuated surface and residual surface for the two DMs. Simulation was ran using a $4096 \times 4096$ size grid

for. In Fig. 3 we show the same simulation ran with a sampling of $\sim 5 \mu \mathrm{m}$. The cut off frequency for the PSD in this case is $\omega_{\text {cutoff }} \approx 1 \times 10^{-4} \mathrm{~nm}^{-1}$. Despite the larger range of frequencies laying outside of the correctible range, the DM is till providing noticeable gain in beam profile uniformity with respect to the uncorrected case (Fig. 1).

\section{A DIFFERENT APPROACH}

In the previous section the two mirrors in the HOMS LCLS beam transport system have been replaced by two deformable mirrors. As already explained the advantage of such a scheme is the fact that no wavefront sensor is needed. An alternative approach would consist of a single DM deployed along an x-ray beamline and used to correct for the wavefront errors produced by conventional optics located upstream of the DM itself. In this section we consider this option. The LCLS transport system is again used as template. The two offset mirrors are placed according to what described in section 2. A a single DM is placed $1.2 \mathrm{~m}$ downstream of the second offset mirror. The DM is defined with specifications matching the DM under development. The figure errors for the offset mirrors are from interferometry data, while the DM has no figure error. The DM surface is actuated in order to reproduce the wavefront that beam would have in the absence of figure errors along the beamline. The grid size used for this simulation limits the cutoff for the PSD at the highest spatial frequency of $\omega_{\text {cutoff }} \approx 6 \times 10^{-5} \mathrm{~nm}^{-1}$. Fig 4 (left panel) shows the results of the simulation. It is important to understand a major difference between the two schemes discussed in section 3 and 4; in the latter the single DM is responsible for correcting wavefront errors introduced upstream by other optics. This implies that, to get optimal wavefront correction, the location 
of the DM along the beamline must be optimize. This can be understood following the formalism of Zhou and Burge. ${ }^{11}$ For each optic they define a sinusoidal figure error $u(x)$ with period $p$ and magnitude $W(z$ is the propagation axis, $x$ is the transverse axis spanning the mirror and wavefront surface):

$$
u_{z=0}(x)=e^{2 \pi W \sin \left(\frac{2 \pi x}{p}\right)}
$$

The authors show how the wavefront error cycles between phase $\left(\psi_{z}\right)$ and amplitude $\left(A_{z}\right)$ error while the beam is propagating downstream:

$$
\begin{aligned}
& \psi_{z}(x)=2 \pi W \cos \left(\frac{2 \pi z}{z_{T}}\right) \sin \left(\frac{2 \pi x}{p}\right) \\
& A_{z}(x)=1+2 \pi W \sin \left(\frac{2 \pi z}{z_{T}}\right) \sin \left(\frac{2 \pi x}{p}\right)
\end{aligned}
$$

The period of these oscillations is the Talbot period $z_{T}=\frac{2 p^{2}}{\lambda}$, where $\lambda$ is the x-ray wavelength. Since our DM can only correct phase errors, its location must coincide with one of the locations along the beamline where the phase error is maximized, at the expense of amplitude errors. In Fig. 4 (right panel) we show the result of our simulation obtained by moving the DM away from the last offset mirror. The dependence of the DM capability to correct wavefront error as a function of position is evident. The analysis is complicate by the fact that figure errors on the optical surface are not limited to a single frequency, producing a range of possible talbot lengths. Further study is needed in order to correctly identify the correct talbot length for the system under study. Nonetheless this preliminary result shows that, if this deployment scheme is chosen, our code will be able to help us identifying the optimal placement location of our DM given the parameters of the beamline of choice.
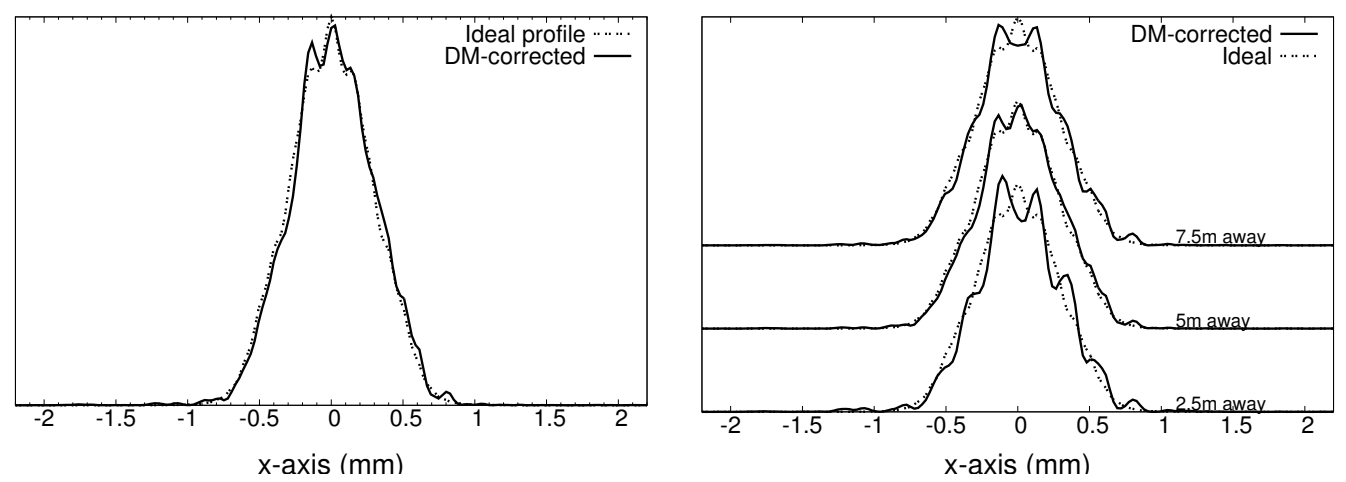

Figure 4. (Left panel): Corrected and diffraction limited beam profile at the LCLS FEH. The DM is placed 5m downstream of the two LCLS offset mirrors. (right panel) The DM location with respect to the last LCLS offset mirrors is varied. Dependence of wavefront correction efficiency on DM location is evident

\section{CONCLUSIONS}

LLNL, in collaboration with Northrop Grumman AOA Xinetics Inc., is currently developing a deformable mirror to be deployed at an x-ray lightsource to achieve wavefront correction in the hard x-ray regime. As part of the project we are also developing code based on wavefront propagation necessary to predict performance of our DM. In this paper we have presented preliminary simulations obtained using our code. The hard x-ray transport system at the Linac Coherent Light Source has been used as template. First we have reproduced results already published in literature, providing a consistency check for our code. Then we discussed the performance of a DM matching the specifications of the DM under development. In a first scenario we replaced the current LCLS offset mirrors with two DMs and commented on the degree of wavefront correction. In a second scenario we deployed the DM downstream of the two LCLS offset mirrors and showed how, due to the Talbot effect, DM placement location is crucial to achieve maximum wavefront correction. These preliminary results show that our code will help us quantify crucial parameters needed for successful deployment of the DM at any x-ray beamline of choice. 


\section{ACKNOWLEDGMENTS}

This work performed under the auspices of the U.S. Department of Energy by Lawrence Livermore National Laboratory under Contract DE-AC52-07NA27344. Document Release Number LLNL-PROC-568453.

\section{REFERENCES}

[1] Emma, P., Akre, R., Arthur, J., Bionta, R., Bostedt, C., Bozek, J., Brachmann, A., Bucksbaum, P., Coffee, R., Decker, F. J., Ding, Y., Dowell, D., Edstrom, S., Fisher, A., Frisch, J., Gilevich, S., Hastings, J., Hays, G., Hering, P., Huang, Z., Iverson, R., Loos, H., Messerschmidt, M., Miahnahri, A., Moeller, S., Nuhn, H., D., Pile, G., Ratner, D., Rzepiela, J., Schultz, D., Smith, T., Stefan, P., Tompkins, H., Turner, J., Welch, J., White, W., Wu, J., Yocky, G., and Galayda, J., "First lasing and operation of an angstrom-wavelength free-electron," Nature Photonics 4, 641 (2010).

[2] Moeller, S., Arthur, J., Brachmann, A., Coffee, R., Decker, F.-J., Ding, Y., Dowell, D., Edstrom, S., Emma, P., Feng, Y., Fisher, A., Frisch, J., Galayda, J., Gilevich, S., Hastings, J., Hays, G., Hering, P., Huang, Z., Iverson, R., Krzywinski, J., Lewis, S., Loos, H., Messerschmidt, M., Miahnahri, A., Nuhn, H.-D., Ratner, D., Rzepiela, J., Schultz, D., Smith, T., Stefan, P., Tompkins, H., Turner, J., Welch, J., White, B., Wu, J., Yocky, G., Bionta, R., Ables, E., Abraham, B., Gardener, C., Fong, K., Friedrich, S., Hau-Riege, S., Kishiyama, K., McCarville, T., McMahon, D., McKernan, M., Ott, L., Pivovaroff, M., Robinson, J., Ryutov, D., Shen, S., Soufli, R., and Pile, G., "Photon beamlines and diagnostics at lcls," Nuclear Instruments and Methods in Physics Research Section A: Accelerators, Spectrometers, Detectors and Associated Equipment 635(1, Supplement), S6 - S11 (2011).

[3] Pivovaroff, M. J., Bionta, R. M., Mccarville, T. J., Soufli, R., and Stefan, P. M., "Soft x-ray mirrors for the linac coherent light source," Advances in X-Ray/EUV Optics and Components II 6705(1), 67050O, SPIE (2007).

[4] McCarville, T. J., Stefan, P. M., Woods, B., Bionta, R. M., Soufli, R., and Pivovaroff, M. J., "Optomechanical design considerations for the linac coherent light source x-ray mirror system," Advances in X-Ray/EUV Optics and Components III 7077(1), 70770E, SPIE (2008).

[5] Soufli, R., Pivovaroff, M. J., Baker, S. L., Robinson, J. C., Gullikson, E. M., Mccarville, T. J., Stefan, P. M., Aquila, A. L., Ayers, J., McKernan, M. A., and Bionta, R. M., "Development, characterization and experimental performance of x-ray optics for the lcls free-electron laser," Advances in X-Ray/EUV Optics and Components III 7077(1), 707716, SPIE (2008).

[6] Soufli, R., Baker, S. L., Robinson, J. C., Gullikson, E. M., McCarville, T. J., Pivovaroff, M. J., Stefan, P., Hau-Riege, S. P., and Bionta, R., "Morphology, microstructure, stress and damage properties of thin film coatings for the lcls x-ray mirrors," Damage to VUV, EUV, and X-Ray Optics II 7361(1), 73610U, SPIE (2009).

[7] Mercere, P., Idir, M., Dovillaire, G., Levecq, X., Bucourt, S., Escolano, L., and Sauvageot, P., "Hartmann wavefront sensor and adaptive x-ray optics developments for synchrotron applications," Adaptive X-Ray Optics 7803(1), 780302, SPIE (2010).

[8] Mimura, H., Handa, S., Kimura, T., Yumoto, H., Yamakawa, D., Yokoyama, H., Matsuyama, S., Inagaki, K., Yamamura, K., Sano, Y., Tamasaku, K., Nishino, Y., Yabashi, M., Ishikawa, T., and Yamauchi, K., "Breaking the 10nm barrier in hard-x-ray focusing," Nat. Phys. 6, 122 (2010).

[9] Krist, J. E., "Proper: an optical propagation library for idl," Optical Modeling and Performance Predictions III 6675(1), 66750P, SPIE (2007).

[10] Barty, A., Soufli, R., McCarville, T., Baker, S. L., Pivovaroff, M. J., Stefan, P., and Bionta, R., "Predicting the coherent x-ray wavefront focal properties at the linac coherent light source (lcls) x-ray free electron laser," Opt. Express 17, 15508-15519 (Aug 2009).

[11] Zhou, P. and Burge, J. H., "Analysis of wavefront propagation using the talbot effect," Appl. Opt. 49, 5351-5359 (Oct 2010). 